\title{
Échapper au piège «qualité-exclusion » dans les indications géographiques : réflexions sur le cas du poivre de Penja
}

\author{
Giovanni Belletti ${ }^{1, *}$, Didier Chabrol $^{2}$ et Greta Spinsanti ${ }^{3}$ \\ ${ }^{1}$ Department of Economics and Management, University of Firenze, Firenze, Italie \\ 2 CIRAD, UMR Innovation, 34398 Montpellier, France \\ ${ }^{3} \mathrm{MSc}$ in Development Economics, University of Firenze, Firenze, Italie
}

\begin{abstract}
Résumé - La protection des indications géographiques (IG) est considérée dans les pays en développement comme une façon de valoriser les produits d'origine et de renforcer les systèmes agroalimentaires localisés en améliorant l'accès au marché des petits producteurs. La démarche d'enregistrement des IG va au-delà de la lutte contre les usurpations des noms géographiques et peut contribuer à modifier le fonctionnement de la filière. En effet, l'enregistrement suppose l'accord des producteurs sur un cahier des charges qui définit les caractéristiques-clés du produit et du processus de production et délimite la zone de production. Cependant, les effets positifs de la protection sont souvent menacés, en particulier dans les pays du Sud, par ce que nous appelons le «piège qualité-exclusion » : la recherche d'une meilleure qualité du produit peut provoquer l'exclusion des producteurs les plus faibles ; à l'inverse, éviter toute exclusion peut conduire à un cahier des charges trop vague ou un système de contrôle trop laxiste, ce qui rendra l'IG inefficace. Nous analysons comment ce dilemme peut être géré pour améliorer l'accès des petits producteurs au marché en examinant le cas du poivre de Penja (Cameroun), enregistré en 2013 et devenu la première IG protégée dans la zone de l'Organisation africaine de la propriété intellectuelle (OAPI). Nos résultats montrent que pour garder tant l'équité que l'efficacité de la protection des IG, il est nécessaire d'accompagner la protection juridique par des actions et des politiques appropriées. Les aspects critiques sont l'élaboration d'un cahier des charges et d'un système de contrôle rigoureux, mais aussi capables de minimiser les règles difficiles à atteindre, et la fourniture de services capables de favoriser l'accès aux IG des producteurs les plus faibles.
\end{abstract}

Mots clés : produits d'origine / accès au marché / petits agriculteurs / action collective / Cameroun

\begin{abstract}
Escaping the "quality-exclusion" trap in building geographical indications: some reflexions from the Penja pepper case. The protection of geographical indications (GI) is considered in developing countries as a powerful tool for supporting origin products and for strengthening local agrifood production systems by improving farmers' market access. GI protection may have several effects that go far beyond the fight against misuses of the geographical name and can stimulate a new organisation and functioning of the supply chain. Indeed, the GI protection requires an agreement of producers on a Code of practices that specifies the production area, the process and product characteristics, and on an appropriate control system. However, GI protection positive effects are often threatened, especially in the context of Southern countries, by what we call "quality-exclusion trap". In fact, searching for a better product qualification can cause the exclusion of less empowered producers. On the contrary, when the Code of practices and the control systems are too flexible, the GI scheme may be inefficient. The paper aims to analyse how the GI quality-exclusion trap could be managed in order to improve market access for small producers. Empirical evidences come from the case of Penja pepper (Cameroon) that obtained the registration as GI in 2013 thus becoming the first protected GI in the African Intellectual Property Organization area. Results highlight that in order to involve less empowered actors and to guarantee equity and efficiency of the GI protection, a set of accompanying actions and policies is required. Crucial issues concern how to fill the gap between the current practices and the rules of the Code of practices, the choices about the control system, and the provision of some public goods and services allowing the weakest actors to benefit of the protected GI as well.
\end{abstract}

Keywords: origin products / market access / smallholders / collective behaviour / Cameroon

\footnotetext{
* Auteur de correspondance : giovanni.belletti@unifi.it
} 


\section{Introduction}

Les produits d'origine sont caractérisés par des attributs de qualité spécifiques résultant de leur lien au terroir, c'est-à-dire de la combinaison étroite de facteurs naturels et humains liés au lieu d'origine du produit (Bérard et Marchenay, 1995, 2004 ; Casabianca et al., 2011 ; Prévost et al., 2014). Aussi sont-ils fréquemment associés avec le nom de ce lieu, l'indication géographique (IG), qui peut devenir objet d'usurpations et d'usages incorrects. Dans la plupart des systèmes juridiques, l'enregistrement des IG comme titres de propriété intellectuelle et la protection légale qui en résulte supposent l'accord d'une organisation représentative des producteurs (et éventuellement des autres acteurs tels que transformateurs, distributeurs, institutions locales, chercheurs) sur un cahier des charges définissant un ensemble de règles communes : caractéristiquesclés du produit et du processus de production et délimitation de la zone de production. Cet accord serait aussi capable de déclencher ou de renforcer la collaboration et la coordination des acteurs dans le système local de production (Fournier, 2008). Une fois que l'IG est enregistrée, le cahier des charges joue le rôle d'une institution au sens de Ménard (2003), c'est-àdire « un ensemble de règles durables, stables, abstraites et impersonnelles » (Ménard, 2003, p. 206) qui nécessitent des dispositifs d'accompagnement pour les rendre effectives, tels que les mécanismes d' " enforcement » légal (application de la protection sur le marché) ou les groupements d'utilisateurs de l'IG (Cañada et Vázquez, 2005 ; Raynaud et al., 2005).

Les systèmes de protection des IG légitiment et signalent la spécificité des produits correspondants, ce qui peut contribuer à améliorer leur commercialisation (Bramley, 2011). Des effets sur le développement territorial peuvent être aussi générés, y compris une augmentation de la valeur ajoutée retenue dans la région de production et une augmentation de la réputation de la région même, avec des effets induits sur le tourisme ou d'autres produits locaux (Suh et MacPherson, 2007). En particulier, la valorisation des produits d'origine au moyen des IG peut permettre la rémunération de ressources territoriales spécifiques, tout en garantissant leur préservation et leur développement, en créant ainsi un cercle vertueux (Vandecandelaere et al., 2009). C'est pour ces raisons que les produits d'origine et les IG sont considérés comme l'un des piliers des systèmes agroalimentaires localisés (SYAL) (Muchnik et al., 2008).

La protection des IG est donc de plus en plus souvent considérée dans les pays en développement comme une façon de valoriser des produits d'origine issus de systèmes productifs composés principalement de petits producteurs souffrant d'une qualification insuffisante de leur produit et de difficultés d'accès au marché (van de Kop et al., 2006 ; Bramley et Bienabe, 2012), en favorisant leur différenciation grâce à l'identité territoriale (Galtier et al., 2013 ; Belletti et al., 2015).

Cependant, les pays en développement font face à bien des difficultés qui réduisent à la fois le nombre des IG protégées par rapport aux potentialités et les avantages qui en résultent pour les producteurs. Ainsi, les effets de la protection des IG pour les petits producteurs sont souvent moindres qu'espéré. Plusieurs problèmes sont signalés dans la littérature, qui dépendent en bonne partie du contenu du cahier des charges et du processus suivi pour le rédiger (Tregear et al., 2007 ; Bowen et Zapata, 2008 ; Galtier et al., 2013).
À travers le cas du poivre de Penja au Cameroun, cet article analyse le processus de construction d'une des premières IG enregistrées en Afrique subsaharienne. Le but est d'identifier les risques et les opportunités présentés par la protection légale des IG pour les initiatives de valorisation et d'amélioration de l'accès au marché des petits producteurs dans le contexte des pays du Sud, et d'analyser les dispositifs d'accompagnement pour limiter ces risques et profiter de ces opportunités.

Nous présentons dans la section suivante le cadre d'analyse. Nous examinons ensuite la filière du poivre de Penja, ses principaux problèmes et le processus de son enregistrement comme IG. Cela nous amènera à discuter les effets et les points critiques de ce processus et enfin, dans la dernière section, à tirer des conclusions générales.

\section{Cadre d'analyse et objectifs}

Dans les pays en voie de développement, de nombreux facteurs peuvent limiter les effets positifs de la protection des IG pour les producteurs petits et moins bien dotés. Les plus importants sont la vulnérabilité économique des petits producteurs et leur faible pouvoir de négociation face aux autres acteurs (grands agriculteurs, transformateurs, commerçants, mais aussi administrations, organismes de recherche...) qui peuvent déséquilibrer le processus de définition des règles communes. L'accès à l'IG et son utilisation effective peuvent alors être difficiles à cause de la distance entre les pratiques courantes (et le niveau de qualité correspondant) et les règles du cahier des charges, ainsi qu'à cause du niveau de formalisation demandé par les plans de contrôle des IG. En effet, l'IG peut aussi être utilisée par les acteurs les plus forts de la filière pour en exclure d'autres ou pour modifier la répartition des bénéfices, en imposant certaines techniques (selon la stratégie du raising rivals' costs observée par Barjolle et Jeanneaux, 2012) ou par la définition des limites géographiques. Par exemple, dans le cas du Queso Chontaleño étudié par Mancini (2013), l'IG a contribué à marginaliser les producteurs les plus pauvres de ce fromage nicaraguayen. Galtier et al. (2013) soulignent les problèmes rencontrés dans la définition de règles efficaces et équitables pour une IG de café en République dominicaine. Bowen et Zapata (2008) montrent que les effets négatifs de la production et transformation de l'agave au Mexique dépendent de la façon dont le cahier des charges de la tequila IG a été rédigé.

Cette situation d'exclusion peut être exacerbée par les acteurs externes qui sont à l'origine des initiatives IG dans le cadre de projets de développement promus par l'État central ou par des organisations étrangères (Raynolds, 2000). Dans des pays où la « culture du terroir » (Bérard et Marchenay, 1995) est très faible, ces acteurs externes peuvent imposer des approches et des valeurs qui ne correspondent pas à la situation locale et ainsi influencer négativement le processus de reconnaissance et ses résultats (Galtier et al., 2013).

D'autres facteurs critiques sont le faible degré d'organisation des producteurs (un des plus importants facteurs de succès des IG pour Barjolle et Sylvander, 2002), et l'inefficacité du secteur public (Biénabe et Marie-Vivien, 2015 ; Quiñones-Ruiz et al., 2016). Dans de nombreux pays, les dispositifs d'accompagnement de l'IG, aussi bien publics que privés, ne sont pas capables de soutenir les démarches légales de reconnaissance, la mise en 
œuvre des procédures de contrôle, et la gestion de l'IG comme un outil de promotion, une fois enregistrée (Quiñones-Ruiz et al., 2015).

Nous proposons ici d'interpréter la difficulté à intégrer les producteurs les plus faibles aux dynamiques et aux bénéfices des IG en termes de piège qualité-exclusion résultant d'une contradiction entre le degré d'efficacité des règles du cahier des charges et le degré de participation des producteurs.

Quand les initiateurs de l'IG (les producteurs, les transformateurs, des organismes d'appui ou d'autres acteurs) conçoivent le cahier des charges comme une institution pour améliorer la qualité du produit afin d'accéder à des opportunités de marché à haute valeur ajoutée, cela suppose de définir des règles exigeantes et de contrôler la conformité des produits, ainsi que d'assurer la traçabilité et la conformité aux normes générales de qualité (par exemple hygiène). Mais cette codification et cette formalisation peuvent avoir pour effet, même en l'absence d'une stratégie visant à exclure, de limiter la participation à l'IG uniquement aux producteurs qui disposent des compétences, équipements et capacités d'organisation nécessaires. Cette focalisation sur les attributs de qualité et de conformité, plutôt que sur les qualités spécifiques liées au terroir, est présente surtout dans les pays en développement et pour les produits qui entrent dans des filières globales, telles que le café et le cacao (Belletti et al., 2015).

À l'opposé, si pour éviter les phénomènes d'exclusion, les acteurs de l'IG prévoient dans le cahier des charges des règles qui ne sont pas assez sélectives, il y a un risque que l'IG soit inefficace, à la fois sur un plan interne (l'amélioration de la qualité n'est pas stimulée, la concurrence déloyale entre producteurs n'est pas combattue) et externe (la qualité n'est pas garantie aux acheteurs, les normes internationales de qualité et de sûreté ne sont pas respectées). Par conséquent, il y a un dilemme entre l'exclusion des producteurs les moins bien pourvus et l'inefficacité de l'initiative IG : dans les deux cas, les effets réels de la protection, en particulier sur les producteurs petits et pauvres, sont moindres que ceux attendus.

Pour analyser la problématique et identifier des solutions possibles pour échapper à ce piège « qualité-exclusion », nous avons étudié le cas d'un produit d'origine du Cameroun, le poivre de Penja, qui est devenu en 2013 la première IG enregistrée par l'Organisation africaine de la propriété intellectuelle (OAPI), qui regroupe 17 États de l'Afrique centrale et occidentale (http://www.oapi.int/).

\section{Méthodes}

La recherche menée sur le poivre de Penja se fonde sur des techniques d'analyse qualitative, à cause du manque de données statistiques sur la culture du poivre (culture marginale au Cameroun) comme sur les effets de l'enregistrement de l'IG (très récent). Nous avons donc focalisé notre attention d'abord sur les dynamiques qui ont conduit à l'approbation du cahier des charges, de façon à mettre en évidence les intérêts et les attentes des différents acteurs, leur niveau de participation, les points critiques de la discussion et les positions des différentes parties prenantes. En deuxième lieu, nous avons analysé la situation et les dynamiques en cours après l'enregistrement, y compris les problématiques concernant le respect du cahier des charges.
La recherche a commencé en 2012 par un séjour de deux mois dans la région de Penja durant les phases finales de définition du cahier des charges du poivre de Penja. Nous avons d'abord examiné les ébauches du cahier des charges et plusieurs documents techniques fournis par Agro-PME (une organisation non gouvernementale locale), l'OAPI et le Centre de coopération internationale en recherche agronomique pour le développement (CIRAD). Nous avons ensuite conduit 41 entretiens semi-structurés sur un échantillon représentatif des acteurs de la filière ( 22 producteurs, 9 pépiniéristes et 10 négociants). Les entretiens semi-structurés, avec quelques questions préparées à l'avance et les autres générées au cours de l'entretien, nous ont permis de mieux saisir les dynamiques en cours, grâce à une interaction et un échange entre le chercheur et l'interrogé (Longhurst, 2003).

La première partie de l'entretien concernait les caractéristiques de l'entreprise, l'activité menée, son rôle à l'intérieur de la filière, les difficultés rencontrées. La deuxième partie était focalisée sur la dynamique IG pour comprendre le rôle de l'acteur dans l'initiative, ses attentes, son niveau de compréhension, ses points de vue sur les règles du cahier des charges. Le choix des acteurs à interviewer était lié aux groupes d'intérêt identifiés dans l'analyse préliminaire de la filière. Pour garantir la représentativité des producteurs, différents critères ont été retenus : zone de provenance, dimensions de l'exploitation, statut à l'intérieur de l'association. Les distributeurs ont été quasiment tous interviewés lors d'une réunion de leur association à Douala. Les pépiniéristes interviewés étaient concentrés dans la zone de Penja, où la plupart exercent. L'enquête a rencontré quelques contraintes : déplacements difficiles, refus de certains acteurs d'être interviewés (surtout distributeurs), difficulté à rencontrer des acteurs non associés.

Enfin, plusieurs interviews en profondeur ont été menées avec les gestionnaires du projet qui ont accompagné le processus d'enregistrement. L'analyse a été mise à jour en 2014 grâce à des entretiens avec quelques témoins privilégiés et à la consultation de documents plus récents.

Une analyse textuelle des entretiens a été menée. Toutes les informations ont été enfin triangulées (Golafshani, 2003) de façon à vérifier leur qualité et pouvoir tirer des conclusions valables.

\section{Résultats}

\subsection{La filière du poivre de Penja : caractéristiques et problèmes}

En Afrique de l'Ouest et du Centre, de nombreux produits agricoles et alimentaires traditionnels sont liés à un territoire spécifique et bénéficient d'une réputation au niveau national et parfois international (Bridier et Chabrol, 2010). Reconnu comme un des meilleurs poivres (Piper nigrum) au monde, le poivre de Penja est l'un d'entre eux. Il est cultivé par des planteurs étrangers depuis le milieu du $\mathrm{xx}^{\mathrm{e}}$ siècle. De nos jours, la culture du poivre est commune dans les districts de Manjo, Loum, Njombé/Penja, Mbanga, Mombo et Tombel, dans le Sud-Ouest du Cameroun, où les sols volcaniques et le climat lui donnent des caractéristiques spécifiques. Presque tout le poivre camerounais y est produit. Comme il s'agit d'une production réduite, il n'y a pas de statistique nationale. Le département du Moungo, où se trouve Penja, est riche de nombreuses 
Tableau 1. Nombre, surface et production des divers types de producteurs (2010).

Table 1. Number, surface and production of various types of producers (2010).

\begin{tabular}{lrccccc}
\hline & $n$ & $\begin{array}{c}\text { Surface } \\
\text { (hectares) }\end{array}$ & $\begin{array}{c}\text { Surface } \\
(\%)\end{array}$ & $\begin{array}{c}\text { Production } \\
\text { (tonnes) }\end{array}$ & $\begin{array}{c}\text { Production } \\
(\%)\end{array}$ & $\begin{array}{c}\text { Rendements } \\
(\mathrm{kg} / \mathrm{ha})\end{array}$ \\
\hline Petits producteurs & 157 & 263 & 55,5 & 11 & 8,1 & 42 \\
Producteurs professionnels & 54 & 157 & 33,1 & 89 & 65,9 & 567 \\
Grande entreprise & 1 & 54 & 11,4 & 35 & 25,9 & 648 \\
Total & 212 & 474 & 100,0 & 135 & 100,0 & 285 \\
\hline
\end{tabular}

Source : élaborations sur données Agro-PME et GRET (2011) et enquête directe.

Tableau 2. Caractéristiques des types de producteurs de poivre de Penja (2011).

Table 2. Characteristics of different types of Penja pepper producers (2011).

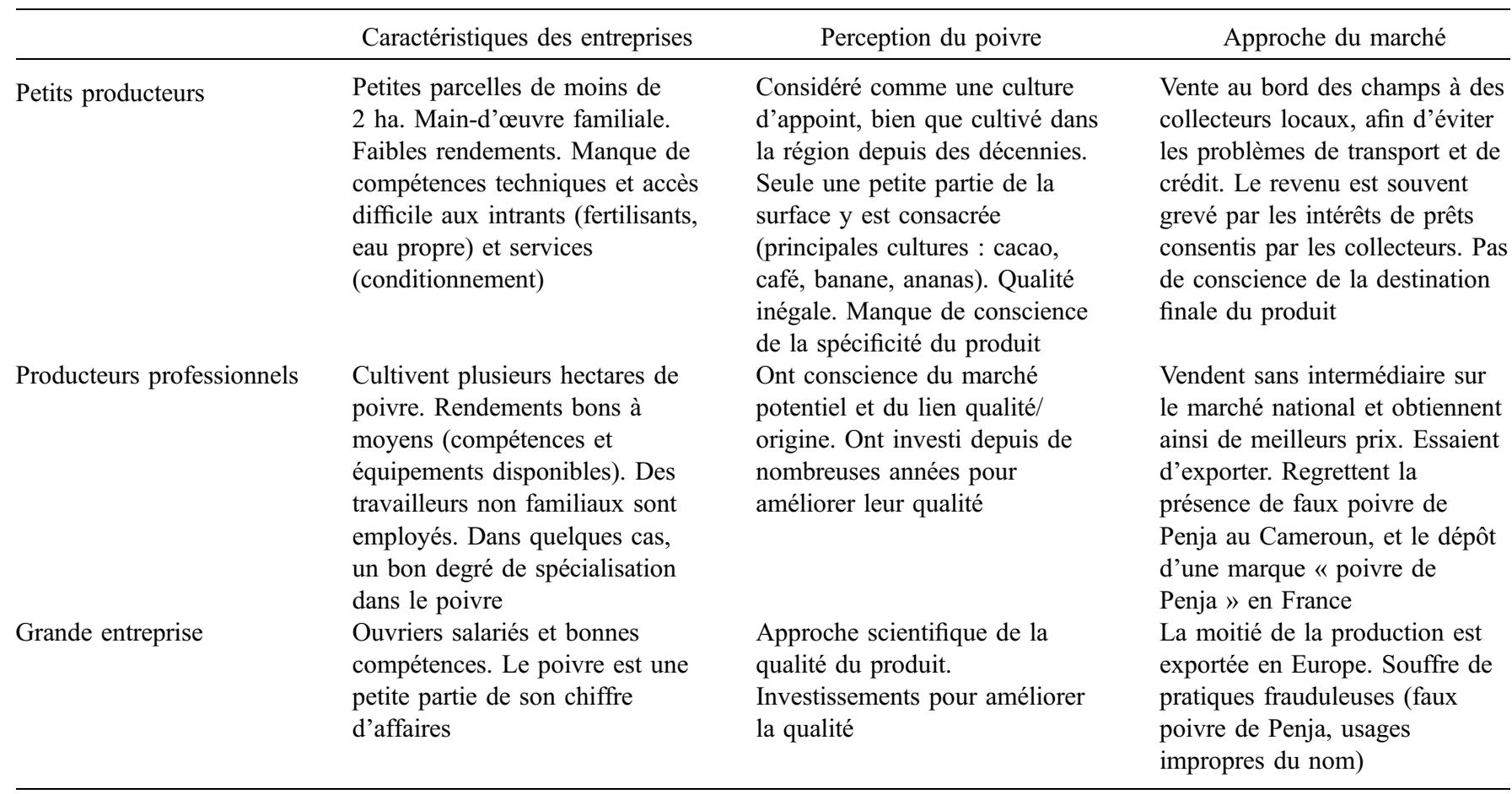

productions : banane, ananas, café, cacao... Le poivre occupe une place marginale dans cette région et ne contribue que pour une faible part aux revenus de la très grande majorité de ses producteurs. Il joue cependant un rôle important pour certains : les «professionnels », mais aussi certains petits agriculteurs pour lesquels une faible contribution au revenu peut faire la différence. Depuis 15 ans, le poivre de cette région est exporté et vendu sur les marchés internationaux en tant que «poivre de Penja », principalement par un négociant français qui l'a lancé sur le marché européen. Au Cameroun même, il est aussi connu comme « poivre de Penja » mais sa vente en vrac rend impossible la garantie de son origine.

Identifié comme IG potentielle dès 2004-2005, le poivre de Penja a été sélectionné en 2008 par le Projet d'appui à la mise en place d'IG (PAMPIG) de l'OAPI, financé par l'Agence française de développement (AFD) avec l'assistance technique du CIRAD. L'objectif général du projet était d'encourager la protection légale des IG dans la zone OAPI où un cadre juridique spécifique était en place depuis 1977. L'implication des petits producteurs et la réduction de la pauvreté étaient aussi visées. En 2011, quand la démarche est entrée dans sa phase active, une étude (Agro-PME et GRET, 2011) a dénombré environ 200 producteurs, une surface cultivée de 474 ha et une production totale de $135 \mathrm{t}$, soit une très faible partie de la production mondiale qui s'établit à plus de $400000 \mathrm{t}$ en 2015. Trois catégories de producteurs ont été identifiées, caractérisées par des structures différentes et confrontées à des opportunités et des difficultés différentes : un grand nombre de petits producteurs; des producteurs professionnels ; et une grande entreprise à capitaux français, la PHP, dont la principale activité est la production de bananes (Tab. 1 et 2). La PHP, filiale de La Compagnie fruitière, s'étend sur 4500 ha et, avec ses 6000 salariés, est le premier employeur privé du pays.

Chez la plupart des producteurs, les rendements sont moyens à très faibles. Cela est principalement dû à la jeunesse 
des plantations mais aussi à des techniques de production inadéquates. Les petits producteurs, en particulier, ne maîtrisent pas certaines techniques (choix des boutures) ou n'ont pas accès à certains intrants (fertilisants, eau propre pour le rouissage). Ni le partage d'informations ni la coordination des acteurs ne sont des pratiques courantes.

Les petits producteurs vendent le poivre directement au bord du champ, à des collecteurs locaux installés sur le marché de Penja, qui fournissent eux-mêmes quelques négociants professionnels installés principalement à Douala. Ceux-ci alimentent le marché national (et quelques pays voisins) à travers des détaillants. Le poivre de Penja est vendu en vrac, sans aucune étiquette. Cette pratique a facilité le développement de conduites frauduleuses : des commerçants mélangent le poivre de Penja avec du poivre importé de moindre qualité, dans le but de vendre le mélange à meilleur marché, ce qui cause une perte de réputation du nom «Penja ». Les producteurs souhaitaient donc plus de garantie sur la qualité et l'origine sur le marché national. Quant au marché international, seule la PHP et quelques producteurs professionnels sont capables d'y accéder. L'importation en Europe est l'exclusivité de fait d'une entreprise française qui a tenté d'acquérir un monopole de droit en enregistrant en France le nom «poivre de Penja» comme marque commerciale, ce qui ne manque pas d'inquiéter les producteurs de Penja. Il existe de nombreux cas d'appropriation sous forme de marque commerciale d'un nom géographique de produit. On peut citer la marque « Parme » enregistrée au Canada, qui interdit au jambon de Parme produit à Parme de porter ce nom au Canada (Thévenod-Mottet et Marie-Vivien, 2011) ou la marque « Rooibos » dont le détenteur aux États-Unis voulait faire payer des royalties aux producteurs sud-africains de Rooibos (Biénabe et Marie-Vivien, 2015).

\subsection{La démarche de protection de I'IG}

En ce qui concerne le poivre, la reconnaissance légale était conçue par le projet PAMPIG et les producteurs comme une stratégie pour améliorer la qualité du produit et mieux le positionner sur les marchés internationaux, et permettre ainsi une meilleure rémunération des producteurs locaux. À cet égard, l'introduction du cahier des charges est significative : l'IG « vise à obtenir un niveau d'excellence pour le poivre de Penja dont les acteurs souhaitent qu'il devienne un des meilleurs poivres au monde et qu'il soit reconnu comme tel ». Il s'agit donc d'améliorer la qualité du produit, également « par l'introduction de mesures nouvelles pour amener davantage de sécurité à la qualité du poivre ainsi que davantage de durabilité au système de production... ».

Depuis le début, les producteurs professionnels ont été les plus impliqués dans la démarche de la protection de l'IG. Ils considéraient principalement la protection comme un moyen de défendre leur produit contre des pratiques commerciales frauduleuses et d'améliorer ainsi leur position dans la filière par rapport aux commerçants. Le soutien actif du projet PAMPIGprincipalement à travers l'ONG Agro-PME - a débuté en 2011 par le soutien à la définition du cahier des charges et à la création de l'association de producteurs. La logique sousjacente au projet PAMPIG était d'inclure les petits producteurs dans l'initiative en organisant leur participation aux discussions sur le cahier des charges et ses règles ainsi que sur le système de contrôle correspondant. Comme prévu dans la méthodologie du projet, qui - en s'inspirant à l'approche FAO-SINERGI telle que présentée par Vandecandelaere et al. (2009) - considérait l'action collective et la coordination entre les acteurs de la filière comme des atouts pour atteindre des effets positifs sur le développement rural durable, une association interprofessionnelle des producteurs du poivre de Penja a été créée en 2011.

Au moment de l'élaboration du cahier des charges, chacune des catégories de producteurs avait des objectifs propres. La grande entreprise, consciente de produire le poivre de la meilleure qualité possible, était soucieuse que les autres producteurs ne tirent pas vers le bas la qualité moyenne. Elle était donc prête à partager les méthodes nécessaires pour obtenir une bonne qualité. Soucieuse de ses bonnes relations avec la société et les institutions locales, elle était également prête à partager les méthodes assurant une bonne productivité. Les petits planteurs, eux, étaient conscients de ne pas maîtriser l'itinéraire de production. La culture du poivre a été introduite dans la région par des planteurs étrangers, et les petits producteurs n'ont jamais bénéficié de mesures d'appui ou de conseil technique. En particulier, beaucoup de plants ne donnaient pas une récolte satisfaisante, même après plusieurs années. Certains planteurs avaient même abandonné la culture. Cette situation était due à une mauvaise maîtrise des techniques de multiplication. Les planteurs professionnels souhaitaient également accéder aux meilleures techniques, afin de pouvoir exporter, mais aussi de constituer un collectif large d'acteurs variés, plus légitime que leurs quelques entreprises.

Il y avait donc une convergence d'objectifs, condition favorable pour une bonne réussite de l'IG. Le projet PAMPIG a donc d'abord demandé à l'agronome consultant de la grande entreprise de rédiger un guide des meilleures pratiques. Ce guide a ensuite été discuté point par point avec des agriculteurs représentatifs des différents types, au cours de trois réunions organisées par Agro-PME fin 2011 et début 2012. Ce processus a permis de faire connaître les techniques recommandées, qui n'étaient pas largement connues jusquelà. Par simplification et modification de certains points, un projet de cahier des charges a été obtenu qui a reçu l'agrément de toutes les parties, y compris la grande entreprise (les petits producteurs n'ont pas été tous consultés, mais seulement un échantillon). Le cahier des charges a également bénéficié des conseils de consultants internationaux, qui n'étaient pas experts du poivre, mais des questions de contrôle, de délimitation ou plus généralement d'IG. L'ensemble de ce processus a duré environ un an.

Certaines dispositions du cahier des charges reflètent la recherche de l'ouverture et manifestent une certaine tolérance :

- la participation à l'association est ouverte à toute personne physique ou morale qui s'engage à respecter le cahier des charges ;

- le matériel végétal est local, facilement accessible à tous ;

- deux méthodes de multiplication sont acceptées : par bouture de racines (la plus complexe, mais qui permet de produire plus de plants dans un délai donné) ou par bouture de tiges (la plus simple, facilement accessible) à condition que les boutures soient prélevées de la partie verte mais ferme de la tige orthotrope d'une plante saine, d'un âge idéal de moins de cinq ans (condition nécessaire à la productivité) ; 
- durant la phase de rouissage, l'eau des bassins doit obligatoirement être changée tous les deux jours (alors que le guide des meilleures pratiques recommandait tous les jours) ;

- le rouissage est aussi possible dans un ruisseau;

- les planteurs ne pouvant avoir accès à des aires cimentées de bonne qualité peuvent sécher leur poivre sur des nattes tissées en fibre végétale, ou sur des bâches plastiques tissées, permettant une circulation de l'air sous les grains. Par contre, les bâches plastiques type film continu sont interdites.

Certaines règles sont d'application différée afin de ne pas constituer un obstacle trop important. Ainsi, ce n'est qu'au 31 décembre 2014 que le taux d'impuretés devait être réduit à $1 \%$ (5\% auparavant), et que les sacs utilisés à la récolte devaient être des sacs neufs réservés à l'usage du poivre (sacs à usage alimentaire auparavant). Quant à l'aire géographique, elle est pour l'instant définie seulement dans ses principes (« l'aire géographique dans laquelle sont réalisées les étapes de production de matière première, de rouissage, de lavage, de séchage et de conditionnement est constituée des territoires des communes de Manjo, Loum, Njombé/Penja, Mbanga, Mombo et Tombel »), et couvre une aire potentielle d'environ $1500 \mathrm{~km}^{2}$ (150 $\left.000 \mathrm{ha}\right)$, c'est-à-dire beaucoup plus que les surfaces actuellement cultivées. En revanche, des conditions spécifiques ont été définies pour identifier les parcelles éligibles à la culture sous IG, comme l'altitude et les sols (les territoires doivent être « situés entre 100 et $500 \mathrm{~m}$ d'altitude et sur soussol basaltique de l'ère quaternaire $»)$, mais l'identification précise des parcelles n'a pas encore été effectuée.

En revanche, certains points sont stricts et reflètent une recherche d'amélioration de la qualité :

- stade de maturité à la récolte (grappes dont quelques grains ont rougi pour le poivre blanc);

- eau propre pour le rouissage ;

- taux de plantation non inférieur à $80 \%$ (par exemple une parcelle d'un hectare aménagée pour 2500 pieds ne devrait pas contenir moins de 2000 plants);

- obligation de conditionner le produit dans la zone, dans le but de faciliter la traçabilité.

Il n'y a pas eu de contradictions fortes au cours de la construction du cahier des charges. On peut y voir la conséquence de la capacité de chacun à faire des concessions, mais aussi la conscience que cette construction pouvait représenter un passage obligé pour obtenir d'autres appuis.

\subsection{Les premiers effets de l'indication géographique}

En 2013, le poivre de Penja a été enregistré en IG par l'OAPI. Une augmentation du prix sur le marché national et à l'exportation a été constatée, du fait de la plus grande visibilité dans les médias conférée par la reconnaissance légale. Dans la période 2010-2013, le prix sur le marché local avait déjà augmenté de 4000 à $7000 \mathrm{FCFA} / \mathrm{kg}$ (environ 6 à $11 €)$, à cause d'une demande croissante stimulée par l'intérêt autour du produit.

Deux ans plus tard, le cahier des charges n'est cependant pas mis en œuvre complètement du fait de difficultés techniques et économiques. Les parcelles satisfaisant au cahier des charges ne sont pas encore identifiées (un projet est en cours en 2015). Le système de contrôle, lui non plus, ne fonctionne pas encore. Selon nos observations, seuls les producteurs professionnels peuvent facilement satisfaire au cahier des charges. Beaucoup de petits producteurs ne maîtrisent pas des facteurs élémentaires tels que la multiplication ou la cueillette à bonne maturité, ne disposent pas d'eau propre pour le rouissage ni d'équipement pour conditionner le produit, et ont du mal à garantir la traçabilité. Cependant, même si l'IG poivre de Penja ne fonctionne pas encore, les effets suivants peuvent être identifiés.

\subsubsection{Intérêt accru et dynamique collective}

Longtemps considérée comme une activité marginale, la production du poivre intéresse de plus en plus les petits producteurs et les commerçants, générant des investissements et des créations d'emplois dans la zone. Le nombre d'acteurs de la filière a été multiplié par 10 en trois ans et la surface cultivée par trois. Du fait de cet intérêt accru et de la diffusion des techniques recommandées par le cahier des charges, la production, le rendement et la qualité du poivre augmentent. L'interaction continue nécessitée par le processus de mise en place de l'IG a conduit les acteurs à se rencontrer, à se connaître et à se reconnaître, facilitant ainsi l'échange d'informations et le partage d'expériences. «L'indication géographique nous a regroupés, finalement on parle la même langue » (interview d'un producteur professionnel).

\subsubsection{Renforcement de la gouvernance de la filière}

L'interaction entre les acteurs a aussi stimulé la naissance d'institutions de gouvernance. Dans le cadre de l'association interprofessionnelle, trois associations professionnelles ont été créées concernant les producteurs, les pépiniéristes, et les marchands et négociants.

\subsubsection{Amélioration de l'accès au marché}

Les associations ont défini des contrats volontaires et collectifs entre les différents maillons de la filière, tels qu'un accord entre producteurs et commerçants pour fixer les prix bord-champ à 7 000/7 500 francs CFA/ $\mathrm{kg}$ en 2013 (environ $11 €$ ) pour garantir un meilleur partage de la valeur ajoutée. Un centre de conditionnement devrait permettre aux petits producteurs d'améliorer leur accès direct au marché et d'augmenter leur pouvoir de négociation.

\subsubsection{Visibilité accrue de la filière poivre au niveau des autorités locales et nationales et mobilisation d'appuis}

Les autorités locales et nationales prêtent maintenant plus d'attention aux projets qui concernent le poivre de Penja. Grâce à l'association interprofessionnelle, les appuis d'ONG, d'établissements bancaires, de l'État et de bailleurs de fonds internationaux ont été mobilisés. On peut citer en particulier : la formation technique des producteurs qui vise l'augmentation de la qualité du poivre et le respect du cahier des charges ; la mise en place d'une formule de micro-crédit aux petits producteurs pour financer l'achat d'engrais (opérationnelle dès 2014) ; la promesse d'investissements de l'État pour améliorer 
la qualité et la quantité de l'eau disponible et les aires de séchage (en cours de réalisation); et la construction grâce à un financement international d'une unité de conditionnement collective permettant aussi d'assurer le contrôle de qualité du produit fini et de fournir aux producteurs des services postrécolte (dont l'entrée en service semble proche). « L'IG nous fera nous développer à travers l'activité économique, nous apportera de nouveaux financements, et de la formation » (interview d'un petit producteur).

\subsubsection{Réappropriation du nom}

Bien que la dénomination "poivre de Penja » ne soit aujourd'hui protégée que dans les 17 pays de l'OAPI, le processus engagé a conduit l'entreprise française dépositaire de la marque en France à ne plus décourager son utilisation par d'autres distributeurs en Europe (Chabrol et al., 2015).

\section{Discussion}

Deux ans après l'enregistrement, bien que l'IG ne soit pas pleinement opérationnelle, celui-ci a déjà produit des effets. Il faudra bien sûr plus de temps pour évaluer les effets spécifiques sur le fonctionnement de la filière, en particulier sur les relations de pouvoir tout au long de celle-ci, sur l'importance des avantages économiques et sur les bénéfices pour les petits producteurs. Cependant, le processus visant à l'enregistrement en IG a clairement activé et catalysé une dynamique locale autour du poivre de Penja (Chabrol et al., 2015), qui concerne aussi les petits producteurs et produit des effets généraux positifs sur le système local de production. On peut en effet repérer plusieurs effets de l'IG qui dépendent plus de la façon dont la démarche d'enregistrement a été gérée que de la protection elle-même, qui d'ailleurs n'est pas valide en dehors des pays membres de l'OAPI. En particulier, une action collective a été initiée, impliquant de nombreux producteurs. Elle a permis le partage et l'internalisation de l'esprit des IG, de ses principes et règles. D'autre part, la visibilité de la démarche IG a stimulé l'intérêt de la presse, des marchés, des investisseurs et des autorités publiques, conformément à la thèse que l'IG n'est pas seulement un outil de défense d'une réputation acquise, mais aussi de construction d'une réputation (Arfini et al., 2011).

Le cas étudié conforte les résultats de Galtier et al. (2013) sur l'importance fondamentale du processus qui mène à la définition des règles communes. Contrairement à ce qui a été décrit par Dentoni et al. (2012) et par Barjolle et Jeanneaux (2012) pour des IG de grande tradition telles que le Prosciutto di Parma (jambon de Parme) AOP et quelques fromages européens en AOP, le processus de rédaction du cahier des charges du poivre de Penja s'est réalisé sans véritables tensions entre les acteurs, et ce malgré la grande hétérogénéité des producteurs concernés en termes de dimension et de capacités. Les acteurs plus puissants et mieux dotés n'ont pas profité de leur position pour exclure les autres ou les asservir à leurs stratégies, contrairement à ce qui avait été constaté dans les dynamiques du café dominicain (Galtier et al., 2013). Cela peut être expliqué par des facteurs généraux et des facteurs contingents au cas étudié.

Parmi les premiers, il faut noter avant tout que la définition des règles du cahier des charges a été basée sur une connaissance préalable des pratiques de production (grâce aux études préliminaires réalisées dans le projet) et une participation de toutes les catégories de producteurs. L'approche suivie a visé non seulement à éviter la condamnation des pratiques traditionnelles mais aussi à permettre l'appropriation par les petits producteurs des pratiques nécessaires à l'amélioration de la qualité. Malgré l'absence d'une réglementation qui oblige à le faire (comme c'est le cas dans quelques pays de l'Union européenne), les différentes parties prenantes ont été incluses dans le processus de rédaction du cahier des charges. Cette participation et une évaluation ex ante des effets potentiels des règles sont considérées comme un préalable à l'utilisation de l'IG par les entreprises après son enregistrement (Belletti et al., 2014). Le fait que le poivre ne soit ni une culture de longue tradition, ni d'une importance fondamentale dans l'économie de la plupart des producteurs de Penja, a facilité un débat et une réflexion plus détendus pour parvenir à un accord partagé. Au lieu de défendre leur propre interprétation du lien au terroir du produit (ce qui se passe souvent en Europe, où le sentiment du « lien au terroir » est plus fort), les producteurs étaient conscients de leurs propres limites techniques. Ils étaient donc disposés à percevoir l'IG (et son cahier des charges) comme un référentiel technique (Filippi et Triboulet, 2006) pour améliorer leur performance productive et pas seulement commerciale. Les producteurs ont donc fait confiance aux acteurs externes à la filière, qui ont joué un rôleclé dans la démarche de la protection de l'IG. Ces acteurs externes ont initié le processus et leur expertise technique et socioéconomique fondée sur des expériences européennes et non européennes a permis de faciliter l'appropriation de la dynamique par les acteurs locaux. D'autre part, l'absence de tradition de débat n'aurait pas été favorable à l'expression forte de désaccords.

L'attitude de la grande entreprise, PHP, constitue un facteur contingent. Elle aurait pu exercer son pouvoir dans la définition du cahier des charges. Au contraire, elle s'est révélée moins intéressée à la rentabilité de la culture du poivre (qui représente une part très réduite de son chiffre d'affaires total) qu'à la qualification du poivre de Penja (qui donne une bonne image de l'entreprise), et surtout qu'à développer des bonnes relations dans le territoire et avec les institutions locales. Elle n'a pas eu une attitude prédatrice ou visant à exclure, mettant au contraire à disposition ses techniciens pour l'élaboration du cahier des charges ainsi que pour fournir de l'appui technique au sein de l'association interprofessionnelle.

Le bon degré d'implication des producteurs dans la dynamique IG dépend aussi des caractéristiques de la filière et du marché du poivre, qui est avant tout un marché régional et national, à la différence de la plupart des autres produits tropicaux qui ont leur marché principal à l'exportation. Cela donne plus d'opportunités d'utilisation directe de l'IG protégée aux petits et moyens producteurs, sans qu'ils doivent affronter les difficultés des marchés internationaux (pouvoir des intermédiaires, manque de protection légale dans certains pays tiers, marques en conflit). Grâce à l'IG, ils ont conscience de pouvoir mieux accéder au marché local et national.

Bien que le cahier des charges du poivre de Penja ne soit pas très sélectif, les producteurs les plus faibles et les plus petits risquent toutefois d'être exclus à cause de leur incapacité à satisfaire aux règles de qualité de base qu'il prévoit. Ainsi, élargir les effets de l'IG poivre de Penja demande des politiques 
d'accompagnement qui sont en cours : soutien à l'organisation interprofessionnelle, formation technique des producteurs, micro-crédit, approvisionnement en eau pour le rouissage, services post-récolte par une unité de conditionnement collective. L'analyse confirme le rôle fondamental des dispositifs d'accompagnement par rapport aux fonctionnements des institutions (le cahier de charges de l'IG) mis en évidence par Ménard (2003). Ce sont pas seulement les dispositifs de contrôle qui sont pertinents (ils ne sont pas encore opérationnels), mais aussi les dispositifs concernant l'appui technique et l'organisation collective, tant avant qu'après l'enregistrement.

Le processus IG a donc contribué à une reterritorialisation de la filière poivre de Penja, au renforcement des liens entre les différents acteurs et bien sûr à l'identification territoriale du produit. Cela permet d'envisager la naissance d'un système agroalimentaire localisé du poivre à Penja, capable de retenir dans le territoire de production une partie croissante de la valeur ajoutée.

\section{Conclusion}

Le piège «qualité-exclusion » caractérise plusieurs initiatives IG, et pas seulement dans les pays en développement. La façon dont le cahier des charges et le système de contrôle sont conçus est d'une importance considérable. Dans le but d'améliorer la qualité du produit d'origine afin de le rendre plus intéressant au point de vue commercial dans le court terme et d'accroître sa réputation dans le long terme, le cahier des charges est souvent strict et bien contrôlé. Mais cela empêche les producteurs petits et faibles de participer à l'IG. Deux solutions sont donc fréquentes : établir des règles faibles qui permettent aux producteurs de continuer à produire et commercialiser comme avant, ou bien écrire un cahier des charges sélectif mais ne pas mettre en œuvre un système de contrôle efficace. Auquel cas l'IG est en fait laissée sans régulation et donc ses effets seront limités.

Le cas du poivre de Penja, bien que l'IG ne soit pas encore en fonction, permet d'identifier un certain nombre de conditions pour échapper au piège " qualité-exclusion ». Ce ne sont pas seulement les règles du cahier des charges qui jouent un rôle fondamental, mais aussi la façon d'arriver à leur définition. Le processus menant à la reconnaissance de l'IG peut en lui-même générer des effets (activation d'une action collective, nouveaux réseaux, innovation...) ou au contraire figer la situation présente de la filière, y compris les déséquilibres de pouvoir et l'exclusion des acteurs les plus faibles. En même temps, le cahier des charges et le système de contrôle doivent être conçus pour inciter les producteurs à améliorer la qualité de leur produit, et les services (tels que la traçabilité et le conditionnement) correspondant aux marchés ciblés doivent être assurés. Cela peut demander un changement de vision de l'IG, conçue non pas comme un outil pour fixer une qualité déjà existante et reconnue par les producteurs en raison d'un lien au terroir qui passe par une tradition (vision méditerranéenne), mais plutôt comme un outil pédagogique capable d'inciter les producteurs à introduire des nouvelles techniques et pratiques et à améliorer la qualité.
Les effets d'une démarche de protection des IG sont plus forts quand elle est intégrée dans une vision plus large. L'IG du poivre de Penja a joué un rôle de catalyseur dans le développement d'une stratégie cohérente autour d'une vision partagée de la qualité du produit. Elle a déclenché l'activation d'importantes innovations qui ouvrent la voie à un fonctionnement plus durable de la filière.

Une stratégie IG proactive et des innovations choisies dans la gouvernance de la filière sont donc d'une importance fondamentale pour surmonter le dilemme entre l'amélioration de la qualité et l'exclusion des petits producteurs. Il faut en particulier considérer les questions critiques suivantes, et y répondre par des actions spécifiques :

- le décalage entre le cahier des charges et les pratiques existantes des producteurs doit être comblé par la formation et la diffusion de techniques adéquates ;

- le degré de précision et de fiabilité des systèmes de contrôle et de traçabilité doit être choisi avec soin, sachant que des contrôles plus stricts supposent des efforts plus importants et des coûts de certification plus élevés, et donc un risque d'exclusion des acteurs les plus faibles ;

- la fourniture de services d'assistance technique et l'appui à des investissements d'intérêt collectif, par l'État ou les associations interprofessionnelles, pouvant permettre aux petits producteurs d'utiliser l'IG.

Des acteurs externes au système productif local, tels que des ONG ou des agences de coopération, peuvent jouer un rôle pertinent pour combler le manque de connaissances et d'expérience dans les pays qui ne sont pas familiers avec le concept d'IG. Ils doivent néanmoins être prudents et veiller à jouer un rôle de soutien et non de substitution aux dynamiques locales. Quand le but est de renforcer le système agroalimentaire local et d'améliorer le sort des producteurs, y compris les plus faibles, l'enregistrement et la protection en IG ne doivent pas être vus comme un pur et simple droit de propriété intellectuelle se suffisant à lui-même, mais plutôt comme un outil parmi d'autres dans le cadre d'une stratégie territoriale plus générale.

Remerciements. Les auteurs remercient le CIRAD pour l'organisation et le financement de la recherche de terrain au Cameroun. Ils remercient aussi M. Metomo Elogo (président du Groupement indication géographique poivre de Penja [GIGPP]), Emmanuel Nzenowo (secrétaire exécutif du GIGPP), Norbert Monkam (directeur général Agro-PME), Cécé Kpohomou (responsable programme IG de l'OAPI) et tous ceux qui ont collaboré aux entretiens.

\section{Références}

Agro-PME, GRET. 2011. Rapport d'étude cartographique des acteurs de la filière poivre de Penja au Cameroun.

Arfini F, Albisu LM, Giacomini C. 2011. Current situation and potential development of geographical indications in Europe. In: Barham E, Sylvander B, eds. Labels of origin for food: local development, global recognition. Wallingford: CABI.

Barjolle D, Jeanneaux P. 2012. Raising rivals costs strategy and localised agro-food systems in Europe. Int J Food Syst Dyn 3(1): 11-21. doi: 10.18461/ijfsd.v3i1.312. 
Barjolle D, Sylvander B. 2002. Some factors of success for origin labelled products in agri-food supply chains in Europe: market, internal resources and institutions. Econ Soc 25(9-10): 45-71.

Belletti G, Brazzini A, Marescotti A. 2014. Collective rules and the use of protected geographical indications by firms. Int Agric Policy 1: $11-20$.

Belletti G, Marescotti A, Touzard JM. 2015. Geographical indications, public goods and sustainable development: the roles of actors' strategies and public policies. World Dev. doi: 10.1016/j.world dev.2015.05.004.

Bérard L, Marchenay P. 1995. Lieux, temps et preuves : la construction sociale des produits de terroir. Terrain Carnets Patrim Ethnol 24: 153-164. doi: 10.4000/terrain.3128.

Bérard L, Marchenay P. 2004. Les produits de terroir. Entre cultures et règlements. Paris: CNRS Editions.

Biénabe E, Marie-Vivien D. 2015. Institutionalizing geographical indications in southern countries: lessons learned from Basmati and Rooibos, World Dev. doi: 10.1016/j.worlddev.2015.04.004.

Bowen S, Zapata AV. 2008. Les appellations d'origine et les durabilités socio-économique et écologique : le cas de la tequila au Mexique. Cah Agric 17(6): 552-560. doi: 10.1684/agr.2008.0241.

Bramley C. 2011. A review of the socio-economic impact of geographical indications: considerations for the developing world. In: WIPO Worldwide Symposium on Geographical Indications, Vol. 22.

Bramley C, Bienabe E. 2012. Developments and considerations around geographical indications in the developing world. Queen Mary J Intellect Prop 1: 14-37. doi: 10.4337/qmjip.2012.01.02.

Bridier B, Chabrol D. 2010. Indications géographiques en Afrique de l'Ouest et du Centre : raisonner la diversité. In: Seiny Boukar L, Boumard P, eds. Savanes africaines en développement : innover pour durer. Actes du colloque, Garoua, Cameroun, 20-23 avril 2009. N'Djamena : PRASAC, 17 p. Disponible sur http://hal.cirad. fr/docs/00/47/43/25/PDF/020 bridier.pdf.

Cañada JS, Vázquez AM. 2005. Quality certification, institutions and innovation in local agro-food systems: protected designations of origin of olive oil in Spain. J Rural Stud 21(4): 475-486. doi: 10.1016/j.jrurstud.2005.10.001.

Casabianca F, Sylvander B, Noël Y, Beranger C, Coulon JB, Roncin F, et al. 2011. Terroir et typicité. Un enjeu de terminologie pour les indications géographiques. In: Delfosse C, ed. La mode du terroir et les produits alimentaires. Paris: Les Indes Savantes.

Chabrol D, Mariani M, Sautier D. 2015. Establishing geographical indications without state involvement? Learning from case studies in Central and West Africa. World Dev. doi: 10.1016/j.world dev.2015.11.023.

Dentoni D, Menozzi D, Capelli MG. 2012. Group heterogeneity and cooperation on the geographical indication regulation: the case of the "Prosciutto di Parma" Consortium. Food Policy 37(3): 207-216. doi: 10.1016/j.foodpol.2012.02.003.

Filippi M, Triboulet P. 2006. Coordination des acteurs et valorisation de produits liés à l'origine. Les signes d'identification comme signes d'exclusion? Rev Econ Reg Urbaine 1/2006: 103-129. doi: 10.3917/reru.061.0103.

Fournier S. 2008. Les indications géographiques: une voie de pérennisation des processus d'action collective au sein des systèmes agroalimentaires localisés ? Cah Agric 17(6): 547-551. doi: 10.1684/agr.2008.0250.

Galtier F, Belletti G, Marescotti A. 2013. Factors constraining building effective and fair geographical indications for coffee: insights from a Dominican case study. Dev Policy Rev 31(5): 597-615. doi: 10.1111/dpr.12027.

Golafshani N. 2003. Understanding reliability and validity in qualitative research. Qual Rep 8(4): 597-606.

Longhurst R. 2003. Semi-structured interviews and focus groups. In: Clifford N, French S, Valentine G, eds. Key methods in geography. London: Sage.

Mancini MC. 2013. Geographical indications in Latin America value chains: a "branding from below" strategy or a mechanism excluding the poorest? J Rural Stud 32: 295-306. doi: 10.1016/j.jrurs tud.2013.07.008.

Ménard C. 2003. L'approche néo-institutionnelle : des concepts, une méthode, des résultats. Cah Econ Polit 44: 103-118. doi: 10.3917/ cep.044.0103.

Muchnik J, Sanz Cañada J, Torres Salcido G. 2008. Systèmes agroalimentaires localisés : état des recherches et perspectives. Cah Agric 17(6): 513-519. doi: 10.1684/agr.2008.0251.

Prévost P, Capitaine M, Gautier-Pelissier F, Michelin Y, Jeanneaux P, Fort F, et al. ( 2014). Le terroir, un concept pour l'action dans le développement des territoires. VertigO - Rev Electron Sci Environ 14(1).

Quiñones-Ruiz XF, Penker M, Vogl CR, Samper-Gartner LF. 2015. Can origin labels re-shape relationships along international supply chains? The case of Café de Colombia. Int J Commons 9(1): 416-439.

Quiñones-Ruiz XF, Penker M, Belletti G, Marescotti A, Scaramuzzi S, Barzini E, et al. 2016. Insights into the black box of collective efforts for the registration of geographical indications. Land Use Policy 57: 103-116. doi: 10.1016/j.landusepol.2016.05.021.

Raynaud E, Sauvee L, Valceschini E. 2005. Alignment between quality enforcement devices and governance structures in the agrofood vertical chains. J Manag Gov 9(1): 47-77. doi: 10.1007/ s10997-005-1571-1.

Raynolds LT. 2000. Re-embedding global agriculture: the international organic and fair trade movements. Agric Hum Values 17(3): 297-309.

Suh J, MacPherson A. 2007. The impact of geographical indication on the revitalisation of a regional economy: a case study of 'Boseong' green tea. Area 39(4): 518-527. doi: 10.1111/j. 1475-4762. 2007.00765.x.

Thévenod-Mottet E, Marie-Vivien D. 2011. Legal debates surrounding geographical indications. In: Barham E, Sylvander F B, eds. Labels of origin for food: local development, global recognition. Wallingford: CABI.

Tregear A, Arfini F, Belletti G, Marescotti A. 2007. Regional foods and rural development: the role of product qualification. $J$ Rural Stud 23(1): 12-22. doi: 10.1016/j.jrurstud.2006.09.010.

van de Kop P, Sautier D, Gerz A, eds. 2006. Origin-labeled products: lessons for pro-poor market development. Amsterdam: KIT and CIRAD.

Vandecandelaere E, Arfini F, Belletti G, Marescotti A. 2009. Linking people, places and products. Rome: FAO/SINERGI.

Citation de l'article : Belletti G, Chabrol D, Spinsanti G. 2016. Échapper au piège « qualité-exclusion » dans les indications géographiques : réflexions sur le cas du poivre de Penja. Cah. Agric. 25: 55002. 